\title{
Formulation Development of Spherical Crystal Agglomerates of Itraconazole for Preparation of Directly Compressible Tablets with Enhanced Bioavailability
}

\author{
Janki Fadke, ${ }^{1}$ Jagruti Desai, ${ }^{1}$ and Hetal Thakkar ${ }^{1,2}$
}

Received 4 April 2015; accepted 8 May 2015; published online 20 May 2015

\begin{abstract}
The objective of the present work was to formulate tablet dosage form of itraconazole with enhanced bioavailability. Spherical crystal agglomerates (SCA) of itraconazole prepared by quasi emulsification solvent diffusion method using Soluplus and polyethylene glycol 4000 (PEG 4000) showed increased solubility $(540 \mu \mathrm{g} / \mathrm{ml})$ in $0.1 \mathrm{~N}$ hydrochloric acid as compared to pure drug $(12 \mu \mathrm{g} / \mathrm{ml})$. A Fourier transform infrared (FTIR) study indicated compatibility of drug with the excipients. The developed SCA were spherical with smooth surface having an average size of $412 \mu \mathrm{m}$. The significantly improved micromeritic properties compared to the plain drug suggested its suitability for direct compression. The antifungal activity of itraconazole was retained in the SCA form as evidenced from the results of the disc diffusion method. The optimized SCA formulation could be easily compressed into tablet with desirable characteristics of hardness $\left(5 \mathrm{~kg} / \mathrm{cm}^{2}\right)$ and disintegration time $(6.3 \mathrm{~min})$. The in vitro dissolution studies showed significant difference in the dissolution profiles of pure drug $(21 \%)$ and SCA formulation $(85 \%)$ which was even greater than that of marketed preparation $(75 \%)$. In vivo pharmacokinetic showed significant enhancement in $C_{\max }$ and $\mathrm{AUC}_{0-\mathrm{t}}$ with relative bioavailability of $225 \%$. The SCA formulation seems to be promising for enhancement of oral bioavailability of itraconazole.
\end{abstract}

KEY WORDS: bioavailability; direct compression; itraconazole; spherical crystal agglomeration.

\section{INTRODUCTION}

Fungal infections (like athlete's foot, finger and toe nail infections, yeast infections, oral thrush, and ringworm) are commonly acquired and are known to persist over time, causing great discomfort (1). There are also systemic and opportunistic fungal infections which can result in more serious diseases, particularly in patients with compromised immune systems $(2,3)$. Itraconazole, a triazole derivative, is used for the treatment of systemic fungal infection and is preferred over other drugs for histoplasmosis, blastomycosis, systemic mycosis, etc. (4). It is a BCS class II drug having low solubility and high permeability. The extremely low solubility results into poor oral bioavailability (55\%) of itraconazole. Presently, it is available in the form of capsule (Sporanox R, Itaspore R, Canditral R) and solution dosage form (Sporanox oral solution). However, the marketed solution dosage form contains high amount of solubility-enhancing agents such as polyethylene glycol (PEG) 20000 and HP- $\beta$-cyclodextrin, which cause osmotic diarrhea (5). It is also available in the form of oral solution at $10 \mathrm{mg} / \mathrm{ml}$. Solutions, in general, are less stable and

\footnotetext{
${ }^{1}$ Pharmacy Department, Faculty of Technology and Engineering, The Maharaja Sayajirao University of Baroda, Vadodara, 390 001Gujarat, India.

${ }^{2}$ To whom correspondence should be addressed. (e-mail: hetal_thakkar11@yahoo.com)
}

difficult to handle as compared to the solid dosage form. Itraconazole is a poorly compressible drug; hence, manufacturing of its tablet dosage form by direct compression is difficult. Onmel (itraconazole) tablets, Merz Pharmaceuticals, are prepared by compression of itraconazole-HPMC solid dispersion obtained by melt extrusion (6), a tedious and time-consuming technique requiring high temperature and pressure (7). In contrast, the manufacturing of tablets by direct compression requires less unit operations and fewer excipients and is a fast, efficient, and cost-effective process in comparison to granulation (8). The present investigation was aimed at the achievement of dual objectives of formulation of a tablet dosage form of itraconazole and enhancement of its bioavailability by increasing its solubility. Various approaches such as addition of directly compressible diluents and use of glidants are used for improvement of flow characteristics and compressibility. Many approaches for enhancement of solubility of poorly soluble drugs such as physical and chemical modifications of drug, particle size reduction, crystal engineering, salt formation, solid dispersion, use of surfactant, and complexation are also reported (9). A simple technique used for increasing both solubility and compressibility of the drugs is spherical agglomeration. Spherical crystallization is a particle design technique, by which crystallization and agglomeration can be carried out simultaneously in one step. It has also been successfully utilized for improvement of flowability and compactability of crystalline drugs (10). It also enables 
coprecipitation of drug and encapsulating polymer in the form of spherical particle. The enhancement of solubility and dissolution of a number of drugs such as aceclofenac (11), gliclazide (12), mebendazole (13), mefenamic acid (14), celecoxib (15), and ibuprofen (16) have been reported using spherical agglomeration technique. So far, there is no work reported whereby enhancement of flowability, compressibility, as well as bioavailability has been achieved using spherical crystal agglomerate (SCA) technique. Hence, the objective of the present investigation was to develop spherical crystal agglomerates of itraconazole with improved flow property, compressibility, and solubility of itraconazole. The prepared spherical crystal agglomerates were compressed into tablets using direct compression method and evaluated for various parameters. In vivo pharmacokinetic studies were performed for the developed tablet formulation, and comparison was done with the marketed capsule preparation.

\section{MATERIALS AND METHODS}

\section{Materials}

Itraconazole was obtained as gift sample from Alembic Ltd., Vadodara, India. Soluplus was kindly gifted by BASF, Mumbai, India. PEG 4000 and starch were purchased from S.D. Fine Chem., Mumbai, India. Dichloromethane was purchased from Spectrochem Pvt. Ltd., Mumbai, India. All other chemicals and reagent used were of analytical grade.

\section{Preformulation Studies}

The saturation solubility, flow property, and compressibility of pure itraconazole were determined during the preformulation studies. Moreover, the drug excipient compatibility was ensured using Fourier transform infrared (FTIR) spectrophotometry.

\section{Saturation Solubility Measurement}

Ten milligrams of itraconazole was weighed accurately and transferred to a 50-ml beaker; $10 \mathrm{ml}$ of $0.1 \mathrm{~N}$ hydrochloric acid was added, and the contents were stirred for $24 \mathrm{~h}$ at room temperature using magnetic stirrer. The sample was centrifuged at $5000 \mathrm{rpm}$ at $4^{\circ} \mathrm{C}$ for $15 \mathrm{~min}$, supernatant was collected, and the absorbance of solution was measured at $255 \mathrm{~nm}$ using $0.1 \mathrm{~N}$ hydrochloric acid as blank. The amount of drug solubilized was calculated by measuring the absorbance of the standard itraconazole solution of a known concentration.

\section{Flow Property and Compressibility (17)}

The various parameters like angle of repose, bulk density, tapped density, Carr index, and Hausner ratio were evaluated for pure itraconazole.

\section{FTIR Study}

Itraconazole, physical mixture of the excipients, and the physical mixture of drug with excipients were separately mixed with potassium bromide at a ratio of $1: 100$, and the pellets were prepared by applying 10 metric ton of pressure using a hydraulic press. The FTIR spectra were recorded for the samples over a range of $4000-400 \mathrm{~cm}^{-1}$ using the FTIR instrument.

\section{Selection and Optimization of Formulation and Process Parameters}

The spherical crystal agglomeration by quasi emulsification technique requires a solvent, an antisolvent, a polymer, and a bridging liquid. For selection of a solvent and an antisolvent, the solubility of itraconazole in various solvents was determined by adding itraconazole in small increments to a known volume of the solvent. The addition was continued till no further itraconazole could be dissolved in the solvent. The amount solubilized was calculated by subtracting the weight of the remaining amount from the initial weight. The solubility $(\mu \mathrm{g} / \mathrm{ml})$ was then calculated by dividing the amount solubilized by the volume of solvent, and the one which showed the highest solubility was selected as a solvent and that which showed lowest solubility was selected as an antisolvent. Bridging liquid was selected on the basis of ability to bridge the quasi emulsion droplets and form spherical crystal agglomerates. Various polymers such as polyvinyl pyrrolidone (PVP), crosslinked PVP, poloxamer 188, PEG 4000, and PEG 6000 were screened for their ability to enhance solubility and improve flow property of itraconazole. The process parameters such as agitation speed, agitation time, and aqueous phase volume were optimized based on the formation of uniform sized discrete spherical agglomerates.

\section{Preparation of SCA}

The SCA of itraconazole were prepared by quasi emulsification solvent diffusion method (18). Soluplus $(0.75 \% \mathrm{w} / \mathrm{v})$ and PEG $4000(0.3 \% \mathrm{w} / \mathrm{v})$ were dissolved in $100 \mathrm{ml}$ distilled water in a $250-\mathrm{ml}$ beaker; $900 \mathrm{mg}$ of itraconazole $(0.9 \% \mathrm{w} / \mathrm{v}$ of aqueous phase) was accurately weighed and dissolved in $5 \mathrm{ml}$ dichloromethane. Aqueous phase was stirred at $700 \mathrm{rpm}$ using a mechanical (propeller) stirrer. Using a glass syringe, the organic phase (drug solution) was added dropwise into the aqueous phase while stirring, which was continued for $15 \mathrm{~min}$; $0.5 \mathrm{ml}$ of dichloromethane was then added as a bridging liquid. The final mixture was continuously stirred for $2 \mathrm{~h}$ until complete evaporation of dichloromethane. This resulted into simultaneous precipitation and spherical agglomeration of itraconazole. The formed spherical crystal agglomerates were filtered using Whatman filter paper, allowed to dry at room temperature, and collected after complete drying for further characterization.

\section{Preparation of Tablet Containing SCA}

Spherical crystal agglomerates equivalent to $200 \mathrm{mg}$ itraconazole were weighed accurately; $20 \mathrm{mg}$ starch, $2 \mathrm{mg}$ talc, and $1 \mathrm{mg}$ magnesium stearate were added and the mixture was taken in a polythene bag and mixed properly for $1 \mathrm{~min}$ to ensure proper mixing. The above mixture was compressed into tablets using a single-punch tablet machine with flat punches. 


\section{Evaluation of SCA}

\section{Saturation Solubility}

Accurately weighed amount of SCA equivalent to $10 \mathrm{mg}$ of itraconazole was transferred to a 50-ml beaker and further procedure was done as described in the preformulation study.

\section{Particle Size, Flow Property, and Compressibility}

The particle size of pure itraconazole and SCA of itraconazole was determined using a microscope at $\times 10$ magnification. The determination of angle of repose, bulk and tapped density, Carr index, and Hausner ratio of the SCA was done as per the procedure described in the preformulation study.

\section{Shape and Morphology}

The shape and surface morphology of the spherical crystal agglomerates were studied using optical microscopy and scanning electron microscope (SEM), respectively. The agglomerates were observed microscopically under a digital microscope (Nikon Digital Sight DS-Fi2) to study their shape. For studying of the surface morphology by SEM, the samples were attached to sample stubs, silver-coated, and viewed using an accelerating voltage at the magnification of $\times 15,000$.

\section{Differential Scanning Calorimetry Study}

The differential scanning calorimetry (DSC) thermograms of itraconazole and SCA form of itraconazole were recorded. Five milligrams of samples were scanned from $20^{\circ} \mathrm{C}$ to $300^{\circ} \mathrm{C}$ under inert nitrogen atmosphere at a heating rate of $10^{\circ} \mathrm{C} / \mathrm{min}$ using a Shimadzu thermal analyzer (Shimadzu DSC-60, TA-60, Japan).

\section{Powder X-ray Diffraction Study}

X-ray diffraction (XRD) study was performed to evaluate changes, if any, in the crystalline nature of the drug. Powder XRD analysis was performed for SCA and pure drug using an X-ray diffractometer (X'pert Pro, PANalytical, Singapore). The samples were irradiated with the monochromatized $\mathrm{CuK} \alpha$ radiation and analyzed between $2^{\circ}$ and $50^{\circ}$ theta.

\section{Antifungal Activity}

In order to ascertain the retention of antifungal activity of itraconazole in its spherical crystal agglomerates form, the developed SCA formulation was tested by agar cup diffusion method (19). The test was carried out using a culture of Aspergillus niger, in potato dextrose agar. Active culture of A. niger strain was inoculated in sterile $0.85 \% \mathrm{NaCl}$ tube in a ratio of 1:9. Further dilution of the culture was done using sterile $0.85 \% \mathrm{NaCl}$ to get $10^{6} \mathrm{CFU} / \mathrm{ml}$. Seeding of culture was performed by swabbing method in which a sterile swab was dipped into the culture suspension and excess fluid removed by pressing gently against the wall of test tube. The swab was placed on the edge of the agar plate and moved across to the other sides. Using a borer, wells of 6-mm diameter were made in the seeded agar plates. Itraconazole plain drug suspension and SCA suspension each containing $100 \mu \mathrm{g}$ itraconazole were added into the wells. Plates were kept in freezer for $15 \mathrm{~min}$ and then in an incubator for a period of $48 \mathrm{~h}$ at $37^{\circ} \mathrm{C}$. After $48 \mathrm{~h}$, the zone of inhibition was measured.

\section{Evaluation of SCA Tablets}

\section{Friability, Hardness, Thickness, and Weight Variation}

The friability of the tablets was determined using the Roche friabilator and \% friability was calculated. Hardness was measured using the Monsanto tablet hardness tester. The thickness of the tablet was measured by using vernier calipers. Weight variation was determined using 20 tablets. Disintegration test was carried out as described under the procedure for uncoated tablet in the Indian Pharmacopoeia (20).

\section{Assay}

The drug content in tablet containing spherical crystal agglomerates was determined. Five tablets were crushed, and accurately weighed powder equivalent to $10 \mathrm{mg}$ of itraconazole was dissolved in $100 \mathrm{ml}$ methanol. The resulting solution was filtered, and after suitable dilution, the absorbance was measured using a UV-vis spectrophotometer. Drug content per tablet was calculated by comparing the absorbance with that of the standard itraconazole solution of known concentration.

\section{In Vitro Dissolution Study}

The USP paddle apparatus II was used for the in vitro dissolution studies; $900 \mathrm{ml}$ of $0.1 \mathrm{~N} \mathrm{HCl}$ was used as the dissolution medium and temperature was maintained at $37^{\circ} \mathrm{C}$ $\pm 0.5^{\circ} \mathrm{C}$ throughout the experiment. In vitro dissolution studies were performed for the following samples: SCA tablet, SCA filled in gelatin capsule, pure itraconazole filled in gelatin capsule, and marketed capsule (Canditral $100 \mathrm{mg}$ ). The samples were placed in separate baskets of the dissolution apparatus. The dissolution apparatus was run at $100 \mathrm{rpm}$, and $5 \mathrm{ml}$ samples were withdrawn at predetermined time intervals $(10$, $20,30,45,60$, and $90 \mathrm{~min}$ ) and replaced with equal volume of the fresh dissolution medium. The samples were filtered, and after suitable dilution, the absorbance was measured using a UV spectrophotometer at $255 \mathrm{~nm}$. Sink conditions were maintained throughout the experiment. The study was carried out in triplicate and mean values were calculated. The amount of the drug dissolved was calculated by comparing the absorbance of the samples with that of standard solution of itraconazole of known concentration.

\section{In Vivo Pharmacokinetic Studies}

Bioavailability studies were performed using male Wistar rats weighing 280 to $350 \mathrm{~g}$. All experiments and protocols described in this study were approved by the Institutional Animal Ethics Committee of The M.S. University of Baroda (Gujarat, India) and were in accordance with guidelines of the Committee for Purpose of Control and Supervision of Experiments on Animals, Ministry of Social Justice and Empowerment, Government of India. Three groups were made for the 
study, and three rats were kept in each group. The animals were kept under standard laboratory conditions, temperature at $25^{\circ} \mathrm{C} \pm 2{ }^{\circ} \mathrm{C}$ and relative humidity $55 \% \pm 5 \%$. The formulations (itraconazole spherical crystal agglomerates in suspended form and extemporaneous suspension prepared from marketed capsule (Canditral $100 \mathrm{mg}$ ) and plain drug suspension) were administered orally using a feeding tube. Dose for the rats was selected as reported and calculated based on the weight of the rats $(30 \mathrm{mg} / \mathrm{kg}$ body weight) according to the surface area ratio. The animals were anesthetized using ether, and blood samples (approximately $500 \mu \mathrm{l}$ ) were collected from the retro-orbital vein using a heparinized needle (18-20 size) at 0 (predose), 1, 2, 4, 6, 8, 10, 12, and $24 \mathrm{~h}$ after oral administration of formulations. The blood samples were collected into a micro centrifugation tube containing $100 \mu l$ of heparin as an anticoagulant, mixed, and centrifuged on a laboratory centrifuge at $5000 \mathrm{rpm}$ for $20 \mathrm{~min}$ at ambient temperature. The supernatant plasma was carefully separated, filled in $1.5-\mathrm{ml}$ micro centrifuge tube, and kept at $-20^{\circ} \mathrm{C}$ until further analysis. Itraconazole detection was performed at $263 \mathrm{~nm}$, using $\mathrm{C}_{18}$ analytical column $(5 \mu \mathrm{m}, 4.6 \times 250 \mathrm{~mm})$. The mobile phase consisted of mixture of water and acetonitrile $(30: 70 \mathrm{v} / \mathrm{v})$ and the flow rate was $1 \mathrm{ml} / \mathrm{min}$. Frozen plasma samples were thawed just prior to extraction; $0.5 \mathrm{ml}$ of plasma sample was transferred to a 2 -ml centrifuge tube. To that, $1.5 \mathrm{ml}$ of acetonitrile was added as a precipitating agent, vortex-mixed for $1 \mathrm{~min}$, and centrifuged at 15,000 rpm for $20 \mathrm{~min}$ at $4^{\circ} \mathrm{C}$. The organic layer was decanted and filtered using syringe filter, and $50 \mu \mathrm{l}$ of the filtrate was injected directly onto the HPLC column. The concentrations were calculated using a calibration plot drawn by analyzing standard itraconazole solutions by the HPLC method described above. Plasma concentrations versus time profile for all the three groups was plotted, and the pharmacokinetic parameters were calculated using Kinetica software (version 5.0).

\section{Stability Studies}

The formulated tablets were subjected to accelerated stability studies. Samples (with blister) were maintained for 6 months in an accelerated stability chamber at $40^{\circ} \mathrm{C} \pm 2^{\circ} \mathrm{C}$ and $75 \% \pm 5 \%$ relative humidity $(\mathrm{RH})(21)$. At intervals of 1 month, the samples were analyzed for drug content and in vitro dissolution rate (in $90 \mathrm{~min}$ ).

\section{RESULTS}

\section{Preformulation Studies}

\section{Saturation Solubility Measurement}

The preformulation studies indicated that itraconazole is having saturation solubility of only $12 \mu \mathrm{g} / \mathrm{ml}$ in $0.1 \mathrm{~N}$ hydrochloric acid.

\section{Flow Property and Compressibility}

The results of the study of flow property and compressibility are shown in Table I.

\section{FTIR Study}

The FTIR spectra of the itraconazole, the physical mixture of the excipients (Soluplus and PEG 4000), and the physical mixture of itraconazole and excipients are shown in Fig. 1. Itraconazole showed peaks at $1184 \mathrm{~cm}^{-1}(\mathrm{C}-\mathrm{N}$ stretching), $1141 \mathrm{~cm}^{-1}$ (C-O-C ring cyclic ether stretching), $1699 \mathrm{~cm}^{-1}$ (conjugated ketone), $1330 \mathrm{~cm}^{-1}$ (aromatic tertiary amine), and $1613 \mathrm{~cm}^{-1}$ (open chain imino $-\mathrm{C}=\mathrm{N}$ - stretching) (22) as can be seen in Fig. 1(a). These peaks are retained in the physical mixture of drug with excipients.

\section{Selection and Optimization of Formulation and Process Parameters}

The optimized parameters for the formation of spherical crystal agglomerates are shown in Table II.

\section{Preparation of SCA and Tablets}

The preparation of spherical crystal agglomerates of itraconazole was done by quasi emulsification solvent diffusion method. Soluplus was used as surfactant in the preparation of the primary emulsion. The formulation parameters were optimized by applying the Box-Behnken design of experiments (data not shown) $(23,24)$. The drug concentrations, Soluplus concentrations, and PEG 4000 were selected as independent variables, while saturation solubility, Carr index, angle of repose, \% yield, and \% drug dissolution were taken as dependent variables. Three factors were evaluated each at three levels, and the experimental trials were performed at all 15 possible combinations given by design. The optimized parameters for the preparation of SCA of itraconazole are shown in Table II. The optimized SCA of itraconazole could be easily compressed into tablets having a smooth surface.

\section{Evaluation of SCA}

\section{Saturation Solubility}

The saturation solubilities of pure itraconazole and optimized SCA formulation in $0.1 \mathrm{~N} \mathrm{HCl}$ were found to be 12 and $540 \mu \mathrm{g} / \mathrm{ml}$, respectively.

\section{Flow Property and Compressibility}

The results of flow property and compressibility study of the SCA are shown in Table I. There is significant improvement in all the flow and compressibility parameters when itraconazole is converted into the SCA formulation.

\section{Particle Size Analysis, Shape, and Morphology}

The particle size of the pure itraconazole powder was found to be $50 \mu \mathrm{m}$ whereas that of the optimized SCA was found to be $412 \mu \mathrm{m}$. The optical microscopic images of pure itraconazole and SCA are shown in Fig. 2a, b, respectively. The SEM images of pure itraconazole and SCA are shown in Fig. 2c, d. The images indicate the conversion of rod-shaped crystals into spherical agglomerates. 
Table I. Results of Different Parameters of Flow Property

\begin{tabular}{lll}
\hline Parameter & Pure itraconazole & SCA of itraconazole \\
\hline Angle of repose $(\theta)$ & 47.46 & $25.54 \pm 0.46$ \\
Bulk density & $0.04 \pm 0.03 \mathrm{~g} / \mathrm{cm}^{2}$ & $0.230 \pm 0.43 \mathrm{~g} / \mathrm{cm}^{2}$ \\
Tapped density & $0.06 \pm 0.06 \mathrm{~g} / \mathrm{cm}^{2}$ & $0.260 \pm 0.56 \mathrm{~g} / \mathrm{cm}^{2}$ \\
Carr index & $33.33 \% \pm 0.14 \%$ & $11.68 \% \pm 0.18 \%$ \\
Hausner ratio & $1.35 \pm 0.42$ & $1.13 \pm 0.25$ \\
\hline
\end{tabular}

$S C A$ spherical crystal agglomerates

\section{DSC Study}

The results of DSC study are shown in Fig. 3. The peak of itraconazole is clear, demonstrating a sharp endotherm at $169^{\circ} \mathrm{C}$ corresponding to its melting point. The DSC thermogram of SCA of itraconazole also shows an endotherm at the same temperature.

\section{XRD Study}

The XRD diffractograms of pure itraconazole and SCA are shown in Fig. 4. The figure shows that the intensity of height of peaks was decreased in SCA of itraconazole (Fig. 4b) in comparison to plain drug (Fig. 4a).

\section{Antifungal Activity}

The mean diameter of zone of inhibition from the plain drug suspension and spherical crystal agglomerates was found to be 14 and $29.5 \mathrm{~mm}$, respectively. Thus, the inhibition of SCA was significantly higher than that of the pure itraconazole.

\section{Evaluation of Tablets}

\section{Friability, Hardness, Thickness, Weight Variation,} Disintegration Time, and Assay

The friability and thickness of prepared tablets were obtained to be $0.73 \%$ and $0.37 \pm 0.12 \mathrm{~cm}$, respectively, which is in the acceptable range. The tablets had sufficient hardness of $5 \mathrm{~kg} /$ $\mathrm{cm}^{2}$ which assures its mechanical strength while handling. The disintegration time was found to be only $6.3 \mathrm{~min}$ which is also acceptable as per various pharmacopeial limits for uncoated tablets. The average weight of 10 tablets was found to be $219.11 \mathrm{mg}$ and none of the tablets showed a variation of more than $4 \%$. The assay was found to be $99.23 \% \pm 0.23 \%$ indicating insignificant loss of drug during preparation.

\section{In Vitro Dissolution Study}

The results of the in vitro dissolution studies are shown in Fig. 5. The \% dissolution of pure itraconazole was found to be

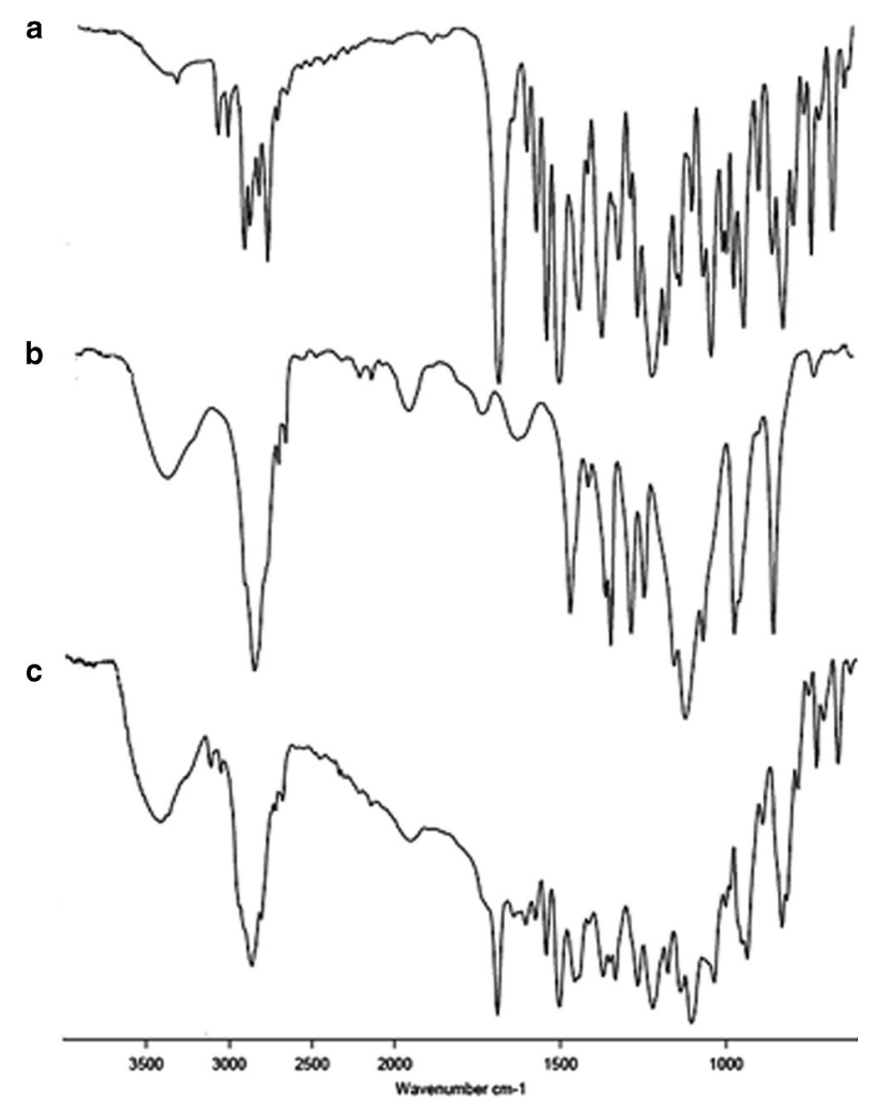

Fig. 1. FTIR spectrums of $a$ itraconazole, $b$ physical mixture of Soluplus and PEG 4000, and $c$ physical mixture of itraconazole, Soluplus, and PEG 4000 
Table II. Optimized Parameters for the Preparation of SCA of Itraconazole

\begin{tabular}{ll}
\hline Parameter & Material/value \\
\hline Good solvent & Distilled water \\
Bad solvent & Dichloromethane \\
Bridging liquid & Dichloromethane $(0.5 \mathrm{ml})$ \\
Polymer & PEG 4000 \\
Agitation speed & $700 \mathrm{rpm}$ \\
Agitation time & $60 \mathrm{~min}$ \\
Aqueous phase volume & $25 \mathrm{ml}$ \\
Drug concentration & $0.9 \% w / v$ of aqueous phase \\
Soluplus concentration & $0.75 \% w / v$ \\
PEG 4000 & $0.3 \% w / v$ \\
\hline
\end{tabular}

$P E G$ polyethylene glycol

$21 \%$ after 90 min and $75 \%$ from marketed capsule. For the SCA formulation, $85 \%$ dissolution was obtained indicating a significant increase $(P<0.05)$ as compared to pure itraconazole and marketed capsule.

\section{In Vivo Pharmacokinetic Studies}

The results of the in vivo pharmacokinetic studies are shown in Table III and Fig. 6. There was a significant enhancement in the $C_{\max }$ and the $\mathrm{AUC}_{0-\mathrm{t}}$ of itraconazole in SCA form in comparison to plain drug suspension and also marketed capsule. The relative bioavailability of the developed formulation was found to be $225 \%$ in comparison to the plain drug suspension. There was 1.56 -fold increase in the bioavailability of itraconazole SCA as compared to marketed capsule.

\section{Stability Studies}

The results of the stability studies (Table IV) indicated that there was no significant change in drug content and \% drug dissolution. Thus, the prepared formulation was stable for a period of 6 months at accelerated conditions.

\section{DISCUSSION}

Spherical crystal agglomeration technique was used in the present investigation for enhancement of solubility and compressibility of itraconazole. It was successfully employed to develop SCA of itraconazole which could be directly compressed into a tablet with enhanced bioavailability.

The principal characteristics required were the enhanced solubility and uniform spherical shape.

An initial screening was done to estimate the solubility of itraconazole obtained after forming SCA using different excipients (data not shown). Soluplus (a polyvinyl caprolactampolyvinyl acetate-polyethylene glycol graft copolymer) (25) was found to give enhanced solubility of itraconazole while PEG 4000 gave spherical shape to agglomerates (observed using optical microscope). Hence, a combination of both these excipients was selected. SCA of itraconazole were prepared by quasi emulsification solvent diffusion method. The quasi emulsion formed contains Soluplus and PEG 4000 in the external aqueous phase. However, these being soluble in
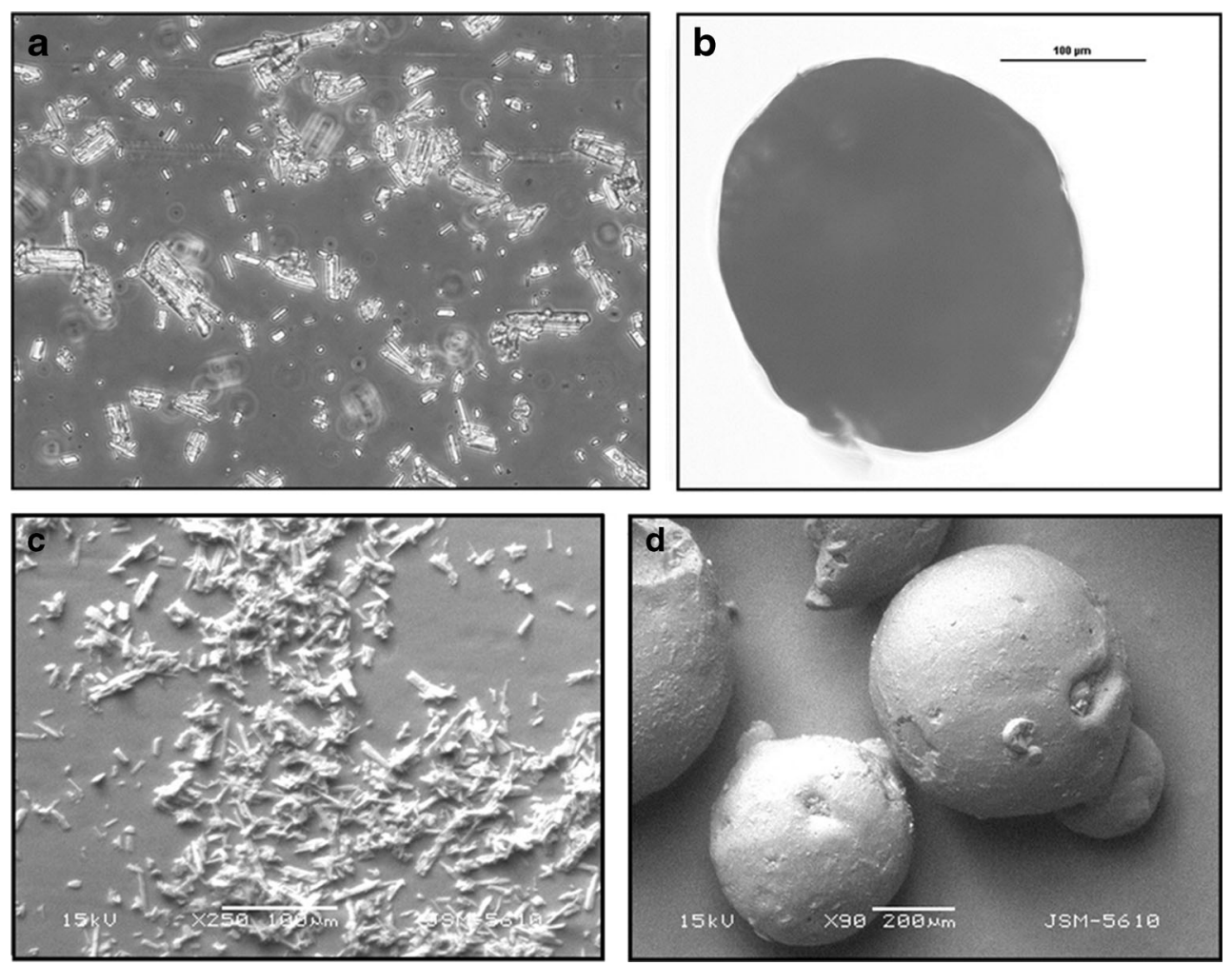

Fig. 2. Optical microscopic images of a itraconazole and $\mathbf{b}$ spherical crystal agglomerates of itraconazole and scanning electron microscopic images of $\mathbf{c}$ itraconazole and $\mathbf{d}$ spherical crystal agglomerates of 

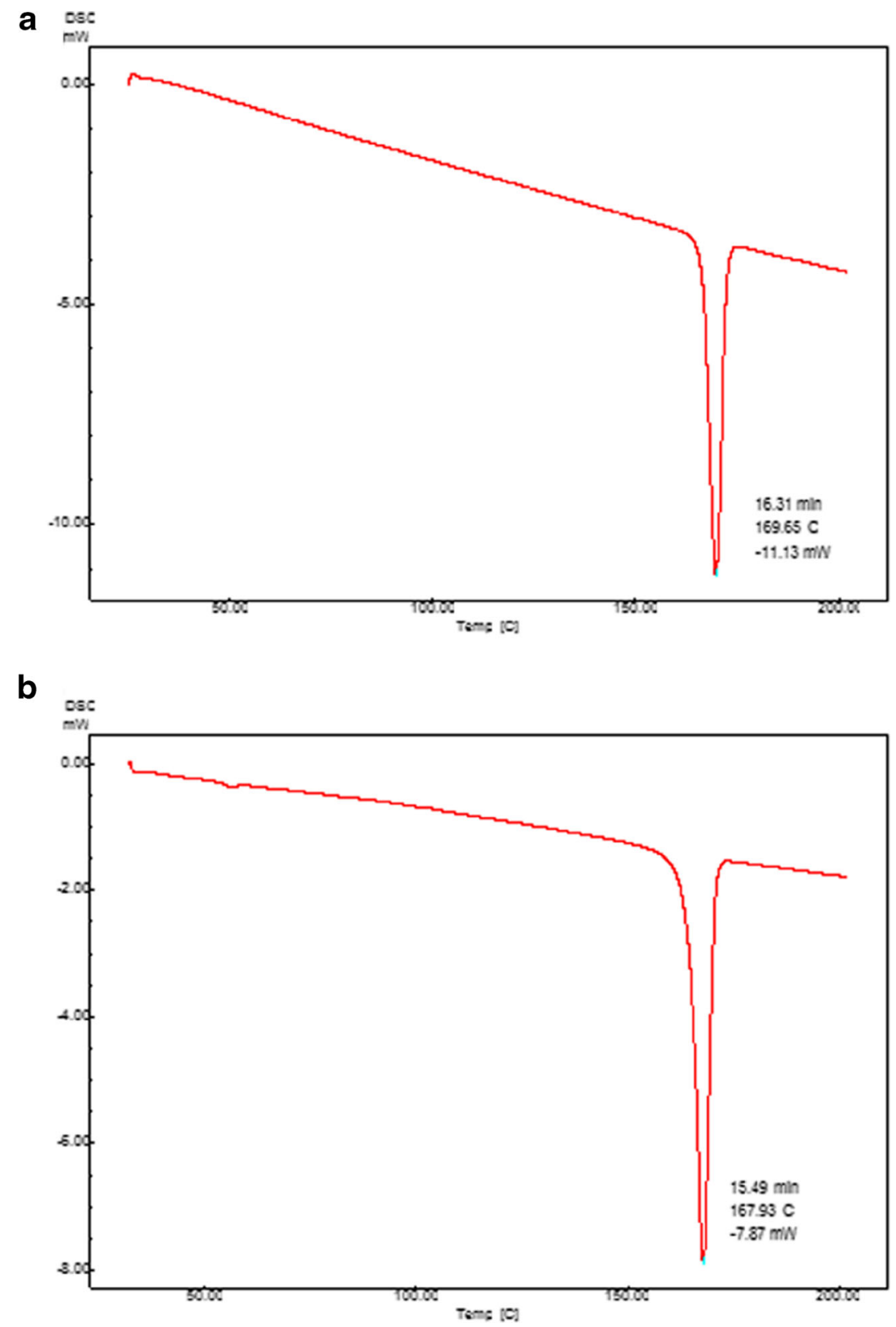

Fig. 3. DSC thermograms of a itraconazole and $\mathbf{b}$ spherical crystal agglomerates of itraconazole

dichloromethane might tend to migrate to the internal organic phase. Upon evaporation of dichloromethane from the spherical emulsion droplets, Soluplus and PEG 4000 may precipitate along with the drug. Soluplus, being polymeric solubilizer, is responsible for enhanced solubility while solidification of PEG 4000 results in formation of SCA with smooth surface. The values of angle of repose (47\%), Carr index (33.3\%), and Hausner ratio (1.65) indicate that itraconazole has extremely poor flow characteristics and compressibility. The SCA formulation showed a significant improvement in all the flow and compressibility parameters indicating its suitability for direct compression. A significant increase in the particle size proves the formation of agglomerates. The improved flow property and compressibility may be attributed to the spherical shape with smooth surface, presence of PEG 4000, and an increase in the size leading to decreased frictional forces. FTIR studies are important in characterizing the interaction between drugs and excipients. There was no significant change in the characteristic peaks of itraconazole in the presence of excipients. This indicated that itraconazole is compatible with Soluplus and PEG 4000. Various methods such as solvent change, quasi emulsification solvent diffusion, and neutralization are reported for preparation of spherical crystal agglomerates. In solvent change method, the drug should be soluble in a watermiscible solvent and neutralization method is more suitable for amphoteric drugs. Itraconazole has poor solubility in water-miscible solvents and thus could not be converted into SCA using solvent change method. Hence, quasi emulsification solvent diffusion method was adopted for preparation of itraconazole SCA. The spherical agglomerates are formed by 

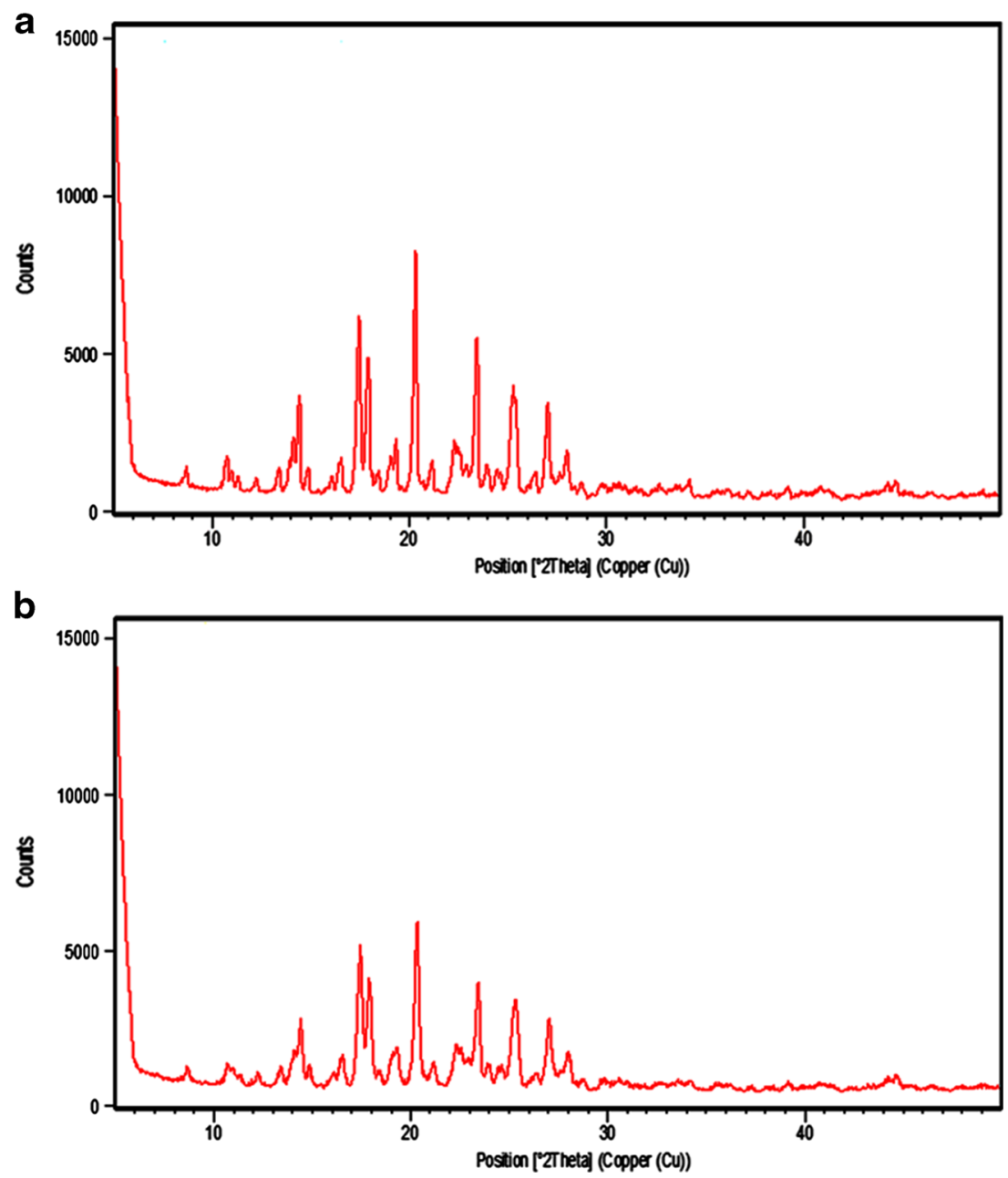

Fig. 4. XRD pattern of $\mathbf{a}$ itraconazole and $\mathbf{b}$ spherical crystal agglomerates of itraconazole

coalescence of the dispersed crystals resulting in an increase in particle size (26). The DSC studies indicate the compatibility of itraconazole upon spherical crystallization using excipients. The significant increase in saturation solubility is attributed to the presence of Soluplus and PEG 4000 apart from its occurrence in the form of spherical agglomerates (27). Moreover, from preliminary studies, it was evident that PEG 4000 did not have any influence on the solubility of itraconazole. It played

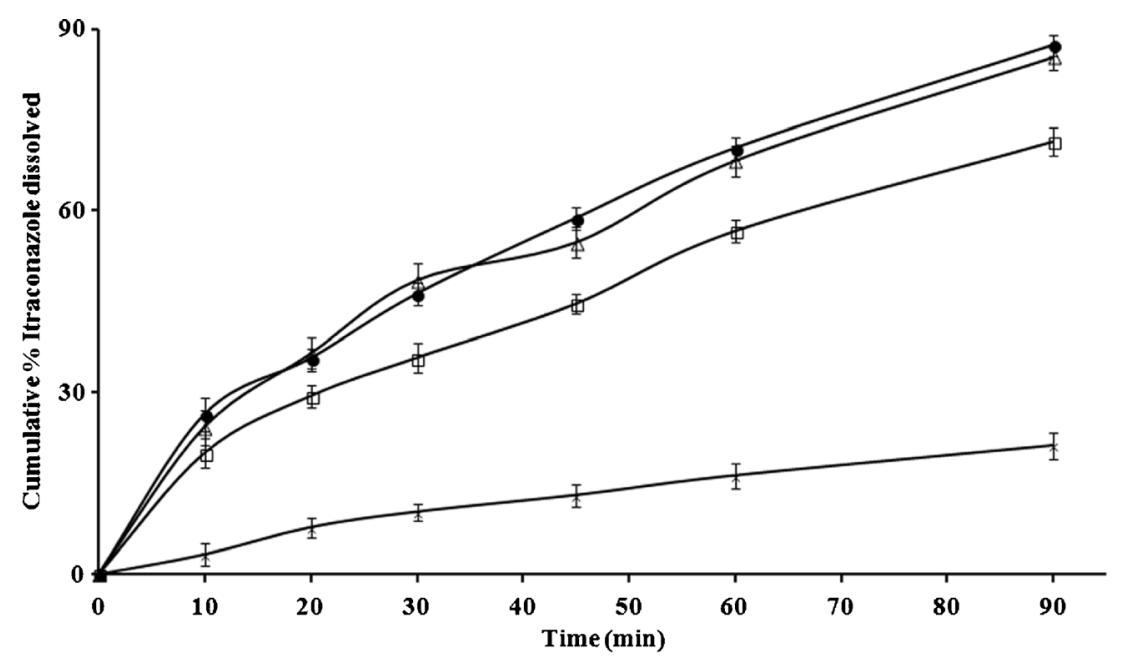

Fig. 5. Cumulative \% itraconazole dissolved in $0.1 \mathrm{~N} \mathrm{HCl}$. SCA of itraconazole filled in capsule (—), marketed capsule of itraconazole $-\square-$, SCA of itraconazole in tablet form $(\neg)$, and pure itraconazole (־). SCA spherical crystal agglomerates 
Table III. Relative Bioavailability and Pharmacokinetic Parameters of Itraconazole After Oral Administration of Itraconazole Plain Drug, Its Spherical Crystal Agglomerates, and Capsule to the Rats $(n=3)$

\begin{tabular}{llll}
\hline Parameters & Plain itraconazole suspension & Spherical crystal agglomerates & Marketed Canditral \\
\hline $\mathrm{AUC}_{0 \rightarrow \mathrm{t}}(\mathrm{ng} / \mathrm{h} / \mathrm{ml})$ & $5504.03 \pm 1.67$ & $12432.71 \pm 1.48$ & $7941.2 \pm 1.23$ \\
$C_{\max }(\mathrm{ng} / \mathrm{ml})$ & 243.83 & $489.99 \pm 1.72$ & $309.58 \pm 0.89$ \\
$T_{\max }(\mathrm{h})$ & $2 \pm 0.3$ & $6 \pm 0.54$ & $6 \pm 0.72$ \\
Relative bioavailability (\%) & - & $225.88 \pm 1.28$ & $144.27 \pm 2.23$ \\
\hline
\end{tabular}

$A U C$ area under the curve

a significant role in obtaining sphericity of the crystals. Soluplus did have a positive influence on the solubility and it increased the solubility of itraconazole around tenfold compared to the pure drug. In general, spherical shape gives higher surface area and wettability to the particles as compared to rod shape of the same size. However, the size of the obtained SCA is significantly higher than that of the rodshaped crystals, and thus, its surface area is considerably reduced compared to the plain itraconazole crystals. Thus, an increase in wettability due to spherical shape and presence of Soluplus seems to be a main factor responsible for enhancement of solubility and dissolution rate. It is evident from the figures that the rod-shaped crystals of itraconazole were agglomerated into spherical shape upon crystal agglomeration. SEM images of pure itraconazole and SCA are shown in Fig. 2c, d. It can be seen that the formulation had a uniform spherical shape and smooth surface which indicated the absence of drug crystals on the surface. The decrease in peak intensity indicates a reduction in the crystallinity of itraconazole in the form of spherical crystal agglomerates. These results are similar with the results obtained by Zhang et al. where itraconazole/Soluplus solid dispersion also showed decreased crystallinity seen in XRD patterns (28). It can be inferred that there is no loss of antifungal activity when itraconazole is converted in to the spherical agglomerate form. In fact, the higher zone of inhibition in case of SCA indicates that due to increased solubility in water, there is availability of higher concentration of drug in the SCA form. Moreover, the concentrations added in the wells were well above the minimum inhibitory concentration of itraconazole which is $1.0245 \mathrm{mg} / \mathrm{l}$ (29). Thus, there was more than fourfold increase in the dissolution of itraconazole. In addition, this higher dissolution within $90 \mathrm{~min}$ is favorable since it is reported that itraconazole absorption is promoted by low stomach $\mathrm{pH}(30)$. There was no significant difference between the dissolution profiles of SCA filled in capsule or compressed in the tablet form $(P>0.05)$. This indicates that the excipients used in the tablet preparation and the compression force used do not affect the dissolution characteristics. In comparison to the marketed capsule also, the spherical crystal agglomerates had significantly higher dissolution rates. The marketed preparation had higher dissolution compared to the plain drug possibly because of the presence of cyclodextrin in it. Further, it is known that cyclodextrin can cause osmotic diarrhea (31), thus limiting its use as an excipient. Hence, the developed SCA formulation which is devoid of cyclodextrin exhibits better dissolution profile compared to the marketed preparation and is expected to enhance the bioavailability (30). It can be inferred from the dissolution studies that in the SCA form, there is almost complete drug dissolution in the gastric conditions within $90 \mathrm{~min}$. Thus, it is expected that the SCA formulation upon administration would have higher bioavailability due to enhanced dissolution during its gastric residence where absorption of itraconazole is reported to be higher as compared to the intestine. The order of dissolution of itraconazole within 90 min was SCA formulation $>$ marketed

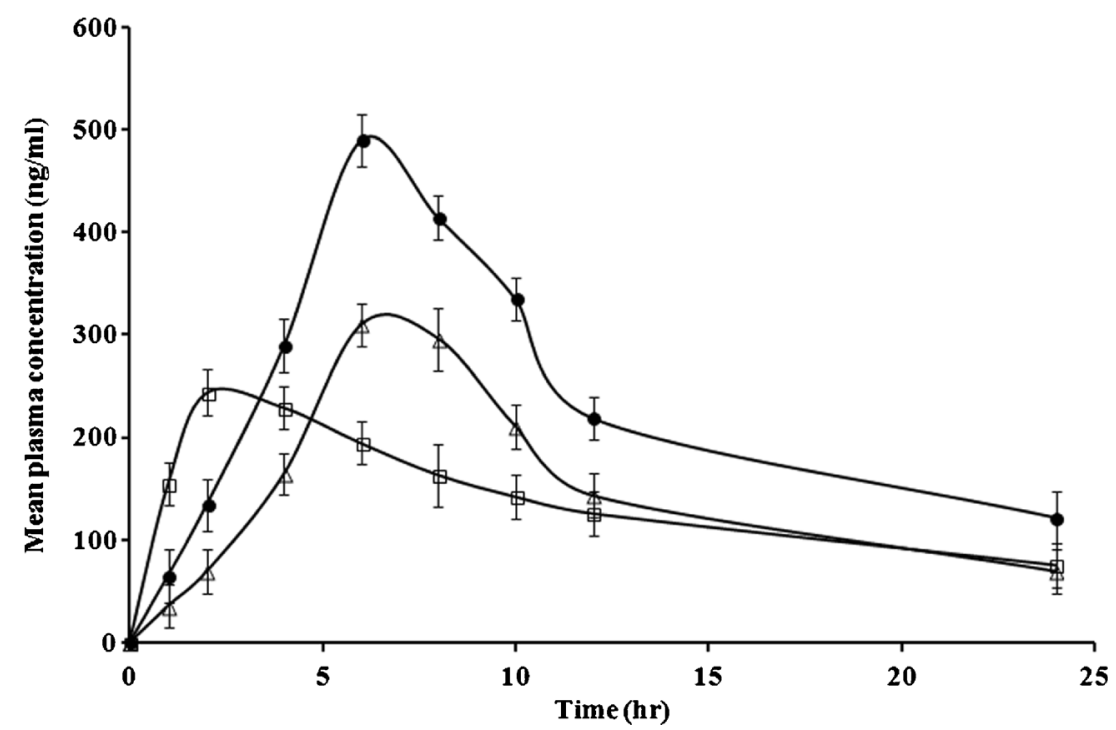

Fig. 6. Concentration versus time curves of itraconazole in plasma medium obtained after administration of plain itraconazole suspension (- - ) , itraconazole SCA $(-\mathbf{C})$, and marketed tablet $(\mathrm{S})$ 
Table IV. Result of Stability Studies of Tablet of Itraconazole SCA at Accelerated Conditions

\begin{tabular}{lll}
\hline & \multicolumn{2}{l}{$\begin{array}{l}\text { Stability study at accelerated condition } \\
40^{\circ} \mathrm{C} \pm 2{ }^{\circ} \mathrm{C} / 75 \% \pm 5 \%\end{array}$} \\
\cline { 2 - 3 } Test after time (month) & $\%$ Drug content & \multicolumn{1}{c}{$\%$ Drug dissolved } \\
\hline Initial & $94.87 \pm 0.81$ & $85.28 \pm 1.23$ \\
1 & $94.21 \pm 1.23$ & $84.79 \pm 0.28$ \\
2 & $94.10 \pm 1.63$ & $84.12 \pm 0.78$ \\
3 & $93.75 \pm 2.1$ & $84.11 \pm 1.4$ \\
\hline
\end{tabular}

$R H$ relative humidity

preparation $>$ plain drug suspension. The same pattern was observed in the values of $C_{\max }$ and AUC confirming the dissolution-limited bioavailability of itraconazole. Moreover, in spite of increase in particle size of itraconazole up on spherical crystallization, there was increase in bioavailability. The reason may be due to the spherical shape of crystals as well as Soluplus. Spherical-shaped particles give better dissolution than irregular-shaped particles. The higher bioavailability, absence of $\beta$-cyclodextrin, and tablet dosage form makes SCA formulation superior over the marketed preparation. The improvement in the oral bioavailability can lead to a decrease in the dose and consequently the side effects associated with the drug.

\section{CONCLUSION}

Spherical crystal agglomeration is a simple technique requiring few excipients as well as instruments. The dual objectives of enhancement of solubility as well as compressibility were achieved with a single formulation. The result of in vivo pharmacokinetic studies correlated well with the in vitro dissolution studies and confirms the dissolutiondependent bioavailability of itraconazole. This was our successful attempt to prepare the tablets of itraconazole by direct compression which is not reported so far. Further research is however advocated for achieving still higher bioavailability to achieve significant dose reduction and consequently side effects.

\section{ACKNOWLEDGMENTS}

The authors acknowledge the TIFAC CORE in NDDS, Government of India, New Delhi, for providing the research facilities to the team.

Conflict of Interest The authors declare that they have no competing interests.

\section{REFERENCES}

1. Hawksworth DL. The magnitude of fungal diversity: the 1.5 million species estimate revisited. Mycol Res. 2001;105:1422-32.

2. Rolando N, Harvey F, Brahm J, Philpott-Howard J, Alexander $\mathrm{G}$, Casewell M, et al. Fungal infection: a common, unrecognised complication of acute liver failure. J Hepatol. 1991;12(1):1-9.
3. Richardson MD. Changing patterns and trends in systemic fungal infections. J Antimicrob Chemother. 2005;56 Suppl 1:i5-11.

4. Porter RS, Kaplan JL, Homeier BP, Albert RK. The Merck manual home health handbook. 3rd ed. Whitehouse Station: Merck Research Laboratories; 2009. xlii, 2306 p., 8 p. of plates p.

5. Rigter IM, Schipper HG, Koopmans RP, Van Kan HJM, Frijlink HW, Kager PA, et al. Relatively bioavailability of three newly developed albendazole formulations: a randomized cross over study with healthy volunteers. Antimicrob Agents Chemother. 2004;1051-1054.

6. Huang Y, Dai W. Fundamental aspects of solid dispersion technology for poorly soluble drugs. Acta Pharm Sin B. 2014;4(1):1825.

7. Yi Y, Yoon HJ, Kim BO, Shim M, Kim SO, Hwang SJ, et al. A mixed polymeric micellar formulation of itraconazole: characteristics, toxicity and pharmacokinetics. J Control Release. 2007;117(1):59-67.

8. Jivraj M, Martini LG, Thomson CM. An overview of the different excipients useful for the direct compression of tablets. Pharm Sci Technol Today. 2000;3(2):58-63.

9. Savjani KT, Gajjar AK, Savjani JK. Drug solubility: importance and enhancement techniques. ISRN Pharm. 2012;2012:195727.

10. Kawashima Y, Imai M, Takeuchi H, Yamamoto H, Kamaiya K, Hino T. Improved flowability and compactibility of spherically agglomerated crystals of ascorbic acid for direct tableting designed by spherical crystallization process. Powder Technol. 2003;130(1):283-9.

11. Usha AN, Mutalik S, Reddy MS, Ranjith AK, Kushtagi P, Udupa N. Preparation, and in vitro, preclinical and clinical studies of aceclofenac spherical agglomerates. Eur J Pharm Biopharm. 2008;70(2):674-83.

12. Varshosaz J, Talari R, Mostafavi SA, Nokhodchi A. Dissolution enhancement of gliclazide using in situ micronization by solvent change method. Powder Technol. 2008;187(3):222-30.

13. Kumar S, Chawla G, Bansal AK. Spherical crystallization of mebendazole to improve processability. Pharm Dev Technol. 2008;13(6):559-68.

14. Viswanathan CL, Kulkarni SK, Kolwankar DR. Spherical agglomeration of mefenamic acid and nabumetone to improve micromeritics and solubility: a technical note. AAPS Pharm Sci Technol. 2006;7(2), E48.

15. Gupta VR, Mutalik S, Patel MM, Jani GK. Spherical crystals of celecoxib to improve solubility, dissolution rate and micromeritic properties. Acta Pharm. 2007;57(2):173-84.

16. Jbilou M, Ettabia A, Guyot-Hermann AM, Guyot JC. Ibuprofen agglomerates preparation by phase separation. Drug Dev Ind Pharm. 1999;25(3):297-305.

17. Lachman L, Lieberman H, Kanig J. The theory and practice of industrial pharmacy. 3 ed. Varghese publishing House. 1987.

18. Pawar AP, Paradkar AR, Kadam SS, Mahadik KR. Crystallo-coagglomeration: a novel technique to obtain ibuprofenparacetamol agglomerates. AAPS Pharm Sci Technol. 2004;5(3), e44.

19. Wagh VD, Deshmukh OJ. Itraconazole niosomes drug delivery system and its antimycotic activity against Candida albicans. ISRN Pharm. 2012;2012:653465.

20. Indian pharmacopoeia. Vol 1. The Indian Pharmacopoeial commission, Government of India, Ghaziabad, 2007: 177.

21. ICH_Guideline. Stability testing of active pharmaceutical ingredients and finished pharmaceutical products, ICH, 2009.

22. Coates J. Interpretation of infrared spectra, a practical approach. In: Meyers RA, editor. Encyclopedia of analytical chemistry. Chichester: John Wiley \& sons Ltd; 2000. p. 10815-37.

23. Singh B, Chakkal SK, Ahuja N. Formulation and optimization of controlled release mucoadhesive tablets of atenolol using response surface methodology. AAPS Pharm Sci Technol. 2006;7(1), E3.

24. Singh B, Rani A, Babita, Ahuja N, Kapil R. Formulation optimization of hydrodynamically balanced oral controlled release bioadhesive tablets of tramadol hydrochloride. Sci Pharm. 2010;78(2):303-23.

25. Technical information. BASF Pharma ingredients and services. http:// www.pharma-ingredients.basf.com/.../EN/.../03_090801e_Soluplus.pdf (2010). Accessed 20 Feb 2014. 
26. Tapas AR, Kawtikwar PS, Sakarkar DM. Enhanced dissolution rate of felodipine using spherical agglomeration with Inutec SP1 by quasi emulsion solvent diffusion method. Res Pharm Sci. 2009;4(2):77-84.

27. Liu C, Liu C, Desai KG. Enhancement of dissolution rate of valdecoxib using solid dispersions with polyethylene glycol 4000. Drug Dev Ind Pharm. 2005;31(1):1-10.

28. Zhang K, Yu H, Luo Q, Yang S, Lin X, Zhang Y, et al. Increased dissolution and oral absorption of itraconazole/Soluplus extrudate compared with itraconazole nanosuspension. Eur J Pharm Biopharm. 2013;85(3 Pt B):1285-92.
29. Kurnatowska A, Kurnatowski P, Horwatt-Bozyczko E, Kurnatowska AJ. Minimal inhibitory concentration (MIC) of caspofungin and itraconazole inhibiting growth of Candida strains calculated from the linear regression equation. Adv Med Sci. 2012;57(1):148-51.

30. Zimmermann T, Yeates RA, Laufen H, Pfaff G, Wildfeuer A. Influence of concomitant food intake on the oral absorption of two triazole antifungal agents, itraconazole and fluconazole. Eur J Clin Pharmacol. 1994;46(2):147-50.

31. Mainous AG, Pomeroy C. Management of antimicrobials in infectious diseases: impact of antibiotic resistance. 2nd ed. New York: Springer; 2010. p. xiv. 412 p. p. 